\title{
Quasiperiodic Envelope Solitons
}

Clausen, Carl A. Balslev; Kivshar, Yuri S.; Bang, Ole; Christiansen, Peter Leth

Published in:

Physical Review Letters

Link to article, DOI:

10.1103/PhysRevLett.83.4740

Publication date:

1999

Document Version

Publisher's PDF, also known as Version of record

Link back to DTU Orbit

Citation (APA):

Clausen, C. A. B., Kivshar, Y. S., Bang, O., \& Christiansen, P. L. (1999). Quasiperiodic Envelope Solitons. Physical Review Letters, 83(23), 4740-4743. https://doi.org/10.1103/PhysRevLett.83.4740

\section{General rights}

Copyright and moral rights for the publications made accessible in the public portal are retained by the authors and/or other copyright owners and it is a condition of accessing publications that users recognise and abide by the legal requirements associated with these rights.

- Users may download and print one copy of any publication from the public portal for the purpose of private study or research.

- You may not further distribute the material or use it for any profit-making activity or commercial gain

- You may freely distribute the URL identifying the publication in the public portal

If you believe that this document breaches copyright please contact us providing details, and we will remove access to the work immediately and investigate your claim. 


\title{
Quasiperiodic Envelope Solitons
}

\author{
Carl Balslev Clausen, ${ }^{1}$ Yuri S. Kivshar, ${ }^{2}$ Ole Bang, ${ }^{1,2}$ and Peter L. Christiansen ${ }^{1}$ \\ ${ }^{1}$ Department of Mathematical Modelling, Technical University of Denmark, DK-2800 Lyngby, Denmark \\ ${ }^{2}$ Australian Photonics Cooperative Research Centre, Research School of Physical Sciences and Engineering \\ Optical Sciences Centre, Australian National University, Canberra ACT 0200, Australia
}

(Received 18 March 1999)

\begin{abstract}
We analyze nonlinear wave propagation and cascaded self-focusing due to second-harmonic generation in Fibbonacci optical superlattices and introduce a novel concept of nonlinear physics, the quasiperiodic soliton, which describes spatially localized self-trapping of a quasiperiodic wave. We point out a link between the quasiperiodic soliton and partially incoherent spatial solitary waves recently generated experimentally.

PACS numbers: 42.65.Tg, 05.45.Yv, 42.65.Ky, 78.66.-w
\end{abstract}

For many years, solitary waves (or solitons) have been considered coherent localized modes of nonlinear systems, with particlelike dynamics quite dissimilar to the irregular and stochastic behavior observed for chaotic systems [1]. However, about 20 years ago Akira Hasegawa, while developing a statistical description of the dynamics of an ensemble of plane waves in nonlinear strongly dispersive plasmas, suggested the concept of an incoherent temporal soliton, a localized envelope of random phase waves [2]. Because of the relatively high powers required for generating self-localized random waves, this notion remained a theoretical curiosity until recently, when the possibility to generate spatial optical solitons by a partially incoherent source was discovered in a photorefractive medium [3], known to exhibit strong nonlinear effects at low powers.

The concept of incoherent solitons can be compared with a different problem: the propagation of a soliton through a spatially disordered medium. Indeed, due to random scattering on defects, the phases of the individual components forming a soliton experience random fluctuations, and the soliton itself becomes partially incoherent in space and time. For a low-amplitude wave (linear regime), spatial incoherence is known to lead to a fast decay. As a result, the transmission coefficient vanishes exponentially with the length of the system, the phenomenon known as Anderson localization [4]. However, for large amplitudes (nonlinear regime), when the nonlinearity length is much smaller than the Anderson localization length, a soliton can propagate almost unchanged through a disordered medium as predicted theoretically in 1990 [5] and recently verified experimentally [6].

These two important physical concepts, spatial selftrapping of light generated by an incoherent source in a homogeneous medium and suppression of Anderson localization for large-amplitude waves in spatially disordered media, result from the effect of strong nonlinearity. When the nonlinearity is sufficiently strong it acts as an effective phase-locking mechanism by producing a large frequency shift of the different random-phase components, and thereby introducing an effective order into an incoher- ent wave packet, thus enabling the formation of localized structures. In other words, both phenomena correspond to the limit when the ratio of the nonlinearity length to the characteristic length of (spatial or temporal) fluctuations is small. In the opposite limit, when this ratio is large, the wave propagation is basically linear.

What will happen in the intermediate case when the length scales of nonlinearity and fluctuations are comparable? It is usually believed that localized structures would not be able to survive such incoherent wave propagation and should rapidly decay. In this Letter we show that, at least for aperiodic inhomogeneous structures, solitary waves can exist in the form of quasiperiodic nonlinear localized modes. As an example, we consider second-harmonic generation (SHG) and nonlinear beam propagation in Fibonacci optical superlattices, and demonstrate numerically the possibility of spatial selftrapping of quasiperiodic waves whose envelope amplitude varies quasiperiodically, while still maintaining a robust, well-defined spatially localized structure, a quasiperiodic envelope soliton.

We consider the interaction of a fundamental wave (FW) with the frequency $\omega$ and its second harmonic $(\mathrm{SH})$ in a slab waveguide with quadratic (or $\chi^{(2)}$ ) nonlinearity. Assuming the $\chi^{(2)}$ susceptibility to be modulated and the nonlinearity to be of the same order as diffraction, we write the dynamical equations in the form

$$
\begin{array}{r}
i \frac{\partial w}{\partial z}+\frac{1}{2} \frac{\partial^{2} w}{\partial x^{2}}+d(z) w^{*} v e^{-i \beta z}=0, \\
i \frac{\partial v}{\partial z}+\frac{1}{4} \frac{\partial^{2} v}{\partial x^{2}}+d(z) w^{2} e^{i \beta z}=0,
\end{array}
$$

where $w(x, z)$ and $v(x, z)$ are the slowly varying envelopes of the FW and $\mathrm{SH}$, respectively. The parameter $\beta=$ $\Delta k\left|k_{\omega}\right| x_{0}^{2}$ is proportional to the phase mismatch $\Delta k=$ $2 k_{\omega}-k_{2 \omega}$, where $k_{\omega}$ and $k_{2 \omega}$ are the wave numbers at the two frequencies. The transverse coordinate $x$ is measured in units of the input beam width $x_{0}$, and the propagation distance $z$ is measured in units of the diffraction length 
$l_{d}=x_{0}^{2}\left|k_{\omega}\right|$. The spatial modulation of the $\chi^{(2)}$ susceptibility is described by the quasi-phase-matching (QPM) grating function $d(z)$. In the context of SHG, the QPM technique is an effective way to achieve phase matching, and has been studied intensively (see Ref. [7] for a comprehensive review).

Here we consider a QPM grating produced by a quasiperiodic nonlinear optical superlattice. Quasiperiodic optical superlattices, one-dimensional analogs of quasicrystals [8], are usually designed to study the effect of Anderson localization in the linear regime of light propagation. For example, Gellermann et al. measured the optical transmission properties of quasiperiodic dielectric multilayer stacks of $\mathrm{SiO}_{2}$ and $\mathrm{TiO}_{2}$ thin films and observed a strong suppression of the transmission [9]. For QPM gratings, a nonlinear quasiperiodic superlattice of $\mathrm{LiTaO}_{3}$, in which two antiparallel ferroelectric domains are arranged in a Fibonacci sequence, was recently fabricated by Zhu et al. [10], who measured multicolor SHG with conversion efficiencies of $\sim 5 \%-20 \%$. This quasiperiodic optical superlattice in $\mathrm{LiTaO}_{3}$ can also be used for efficient direct third-harmonic generation [11].

The quasiperiodic QPM grating is the same as the one fabricated and studied by Zhu et al. [10]. It has two building blocks $A$ and $B$ of length $l_{A}$ and $l_{B}$, respectively, which are ordered in a Fibonacci sequence according to the production rule $S_{j}=S_{j-1} \mid S_{j-2}$ for $j \geq 3$, with $S_{1}=A$ and $S_{2}=A B$ [Fig. 1(a)]. Each block has a domain of length $l_{A_{1}}=l\left(l_{B_{1}}=l\right)$ with $d=+1$ (shaded) and a domain of length $l_{A_{2}}=l(1+\eta)\left[l_{B_{2}}=l(1-\tau \eta)\right]$ with $d=-1$ (white). In the case of $\chi^{(2)}$ nonlinear QPM superlattices this corresponds to positive and negative ferroelectric domains, respectively. For our simulations presented below we have chosen $\eta=2(\tau-1) /\left(1+\tau^{2}\right)=0.34$, where $\tau=(1+\sqrt{5}) / 2$ is the so-called golden ratio. This means that the ratio of length scales is also the golden ratio, $l_{A} / l_{B}=\tau$. Furthermore, we have chosen $l=0.1$. The grating function $d(z)$ varies between +1 and -1 according to the Fibonacci sequence and has the Fourier series

$$
d(z)=\sum_{m, n} d_{m, n} e^{i G_{m, n} z}, \quad G_{m, n}=\frac{2 \pi(m+n \tau)}{D},
$$

(a)

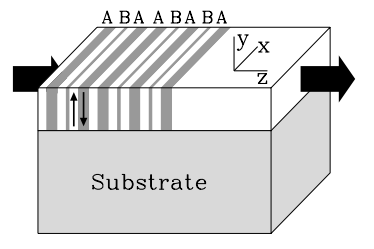

(b)

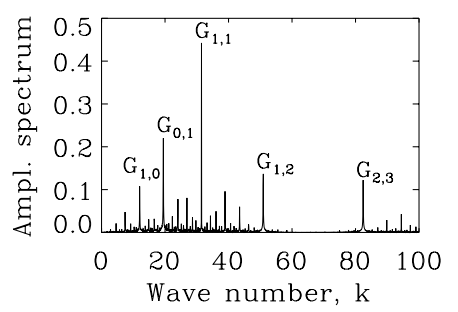

FIG. 1. (a) Slab waveguide with quasiperiodic QPM superlattice structure composed of building blocks $A$ and $B$. (b) Numerically calculated amplitude spectrum of $d(z)$. where $D=\tau l_{A}+l_{B}=0.52$ for the chosen parameter values. Hence the spectrum is composed of sums and differences of the basic wave numbers $\kappa_{1}=2 \pi / D$ and $\kappa_{2}=$ $2 \pi \tau / D$. These components fill the whole Fourier space densely, since $\kappa_{1}$ and $\kappa_{2}$ are incommensurate. Figure 1(b) shows the numerically calculated Fourier spectrum $G_{m, n}$. The lowest-order "Fibonacci modes" are clearly the most intense. From Eq. (2) and the numerically found spectrum, we identify the six most intense modes presented in Table I. The corresponding wave numbers $G_{m, n}$ are in good agreement with Eq. (2).

To analyze the beam propagation and SHG in a quasiperiodic QPM grating, one could simply average Eqs. (1). To lowest order this approach always yields a system of equations with constant mean-value coefficients, which does not allow one to describe oscillations of the beam amplitude and phase. However, here we wish to go beyond the averaged equations and consider the rapid largeamplitude variations of the envelope functions. This can be done analytically for periodic QPM gratings [12]. However, for the quasiperiodic gratings we have to resolve to numerical simulations.

Thus we have solved Eqs. (1) numerically with a second-order split-step routine, in which the linear part is solved with the fast-Fourier-transform method and the nonlinear part is solved with a fourth-order Runge-Kutta scheme. The step length adapts to the local domain length of the QPM grating. At the input of the crystal we excite the fundamental beam (corresponding to unseeded SHG) with a Gaussian profile,

$$
w(x, 0)=A_{w} e^{-x^{2} / 10}, \quad v(x, 0)=0 .
$$

We consider the quasiperiodic QPM grating with matching to the peak at $G_{2,3}$, i.e., $\beta=G_{2,3}=82.25$.

The all-important length scales are the scale of the quasiperiodic fluctuations, which is $l=0.1$, and the nonlinearity length, which is roughly 1 over the input peak intensity, i.e., $L=1 / A_{w}^{2}$. First, we study the smallamplitude limit when a weak FW is injected with amplitude $A_{w}=0.25$. Figures 2(a) and 2(b) show an example of the evolution of the FW and SH in this effectively linear regime, where the fluctuation scale $l=0.1$ is much shorter than the nonlinearity length $L=16$. We see that the SH is excited, but fluctuations and diffraction prevail so that both the FW and SH eventually spread out.

Figures 2(c) and 2(d) show an example of the evolution of a strong input FW beam with $A_{w}=5$, and its corresponding SH. Again the $\mathrm{SH}$ is generated, but now the nonlinearity is strong enough to dominate the fluctuations, $L=0.04<l$. This leads to self-focusing and mutual

TABLE I. The six most intense Fibonacci modes $G_{m, n}$.

\begin{tabular}{ccccccc}
\hline \hline$m$ & 1 & 0 & 1 & 2 & 1 & 2 \\
$n$ & 1 & 1 & 2 & 3 & 0 & 4 \\
$G_{m, n}$ & 31.42 & 19.42 & 50.83 & 82.25 & 12.00 & 101.66 \\
\hline \hline
\end{tabular}



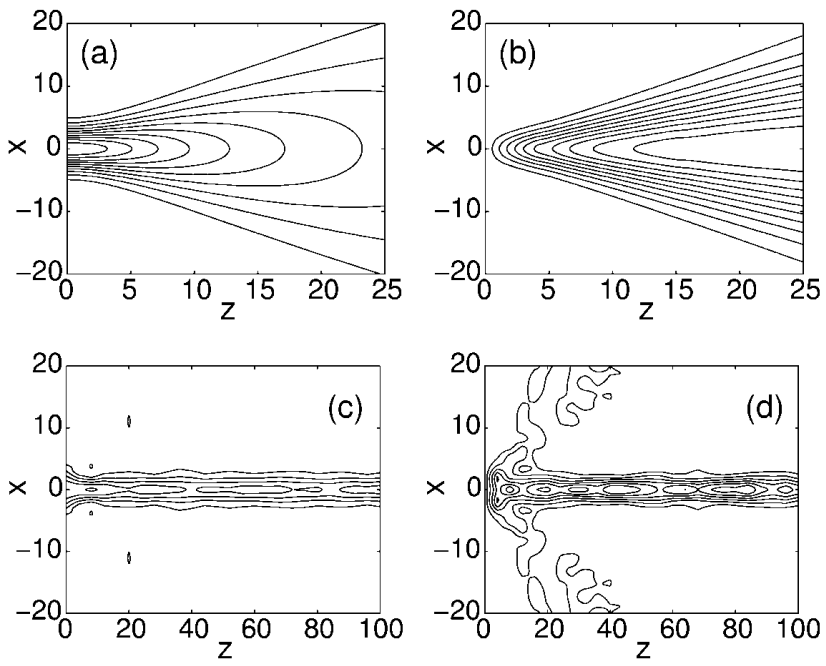

FIG. 2. (a) Diffraction of a weak FW beam with amplitude $A_{w}=0.25$ for $\beta=82.25$. (c) Excitation of a quasiperiodic soliton by a FW beam with amplitude $A_{w}=5$ for $\beta=82.25$. (b),(d) Corresponding SH components.

self-trapping of the two fields, and as a result a spatially localized two-component soliton is generated after an initial transient. The soliton continuously emits radiation due to the scattering off the quasiperiodic QPM grating, but due to the strong self-trapping effect of the nonlinearity the radiation is extremely weak and the soliton remains nicely localized for more than a hundred diffraction lengths. In typical experiments a stable spatial soliton is said to be observed if it remains localized for about 5-10 diffraction lengths. Thus, for all practical purposes, the soliton generated here is dynamically stable.

It is important to notice that the generated twocomponent soliton is itself quasiperiodic. As a matter of fact, after the initial transient, its amplitude oscillates in phase with the quasiperiodic QPM modulation $d(z)$. This is illustrated in Fig. 3, where we show in more detail the peak intensities in the asymptotic regime of the evolution.

Since the oscillations shown in Fig. 3 are in phase with the oscillations of the QPM grating $d(z)$, their spectra should be similar. This is confirmed by Fig. 4, which gives the spectrum of the peak intensity $|w(0, z)|^{2}$ of the
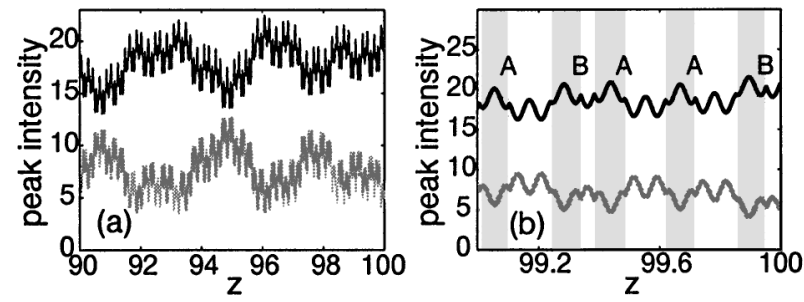

FIG. 3. Amplitude oscillations of the quasiperiodic soliton. (a),(b) Close-ups of the peak intensity $|w(0, z)|^{2}$ of the FW (black) and $|v(0, z)|^{2}$ of the SH (grey). The Fibonacci building blocks $A$ and $B$ are indicated in (b) with $d=1$ in grey regions and $d=-1$ in white regions.
FW. Note that the Fibonacci peak at $k=82.25$ is suppressed (or reduced) because the identical mismatch $\beta$ down-converts it to the dc component. Sum and difference wave numbers between $\beta$ and $G_{m, n}$ appear, which are generated by the nonlinearity. For example, the component at $k=62.8$ is the difference between $\beta=82.25$ and $G_{0,1}=19.42$.

Our numerical results show that the quasiperiodic envelope solitons can be generated for a broad range of the phase mismatch $\beta$. The amplitude and width of the solitons depend on the effective mismatch, which is the separation between $\beta$ and the nearest strong peak $G_{m, n}$ in the Fibbonacci QPM grating spectrum. Thus, low-amplitude broad solitons are excited for $\beta$ values in between peaks, whereas high-amplitude narrow solitons are excited when $\beta$ is close to a strong peak, as shown in Figs. 2(c) and 2(d).

The existence of spatially localized self-trapped states in nonlinear quasiperiodic media should not depend on the particular kind of nonlinearity. The dependence on $\beta$ observed here for the $\chi^{(2)}$ gratings is simply because the "real" strength of the $\chi^{(2)}$ nonlinearity is inversely proportional to the phase mismatch. In fact, it is well known that for large values of the mismatch $\beta$ the quadratic nonlinearity becomes effectively cubic [13]. Thus, our findings are directly applicable to nonlinear optical superlattices in cubic (or $\chi^{(3)}$ ) nonlinear media.

To analyze in more detail the transition between the linear (diffraction) and nonlinear (self-trapping) regimes, we have made a series of careful numerical simulations. In Fig. 5 we show the transmission coefficients and the beam widths at the output of the crystal versus the intensity of the FW input beam, for a variety of $\beta$ values. The input intensities cover the regime of interest, in which the nonlinearity length scale ranges from being much longer than the fluctuation length scale $L=\infty \gg l$ to being shorter than the fluctuation scale $L=0.03<l$. The observed dependencies clearly illustrate the universality of

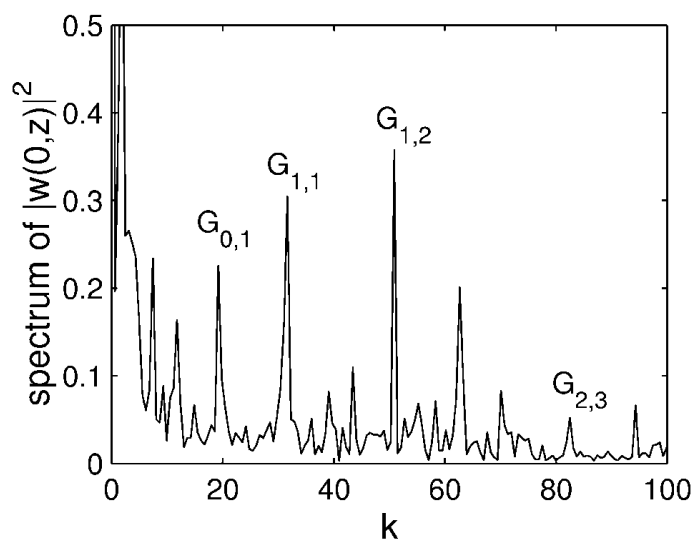

FIG. 4. Spectrum of the amplitude oscillations of the FW component of the quasiperiodic soliton, calculated from $z=90$ to $z=100$ in Fig. 3(a). The peaks correspond to the Fibonacci peaks $G_{m, n}$ in $d(z)$ and sum and difference thereof with the mismatch $\beta=82.25$. 

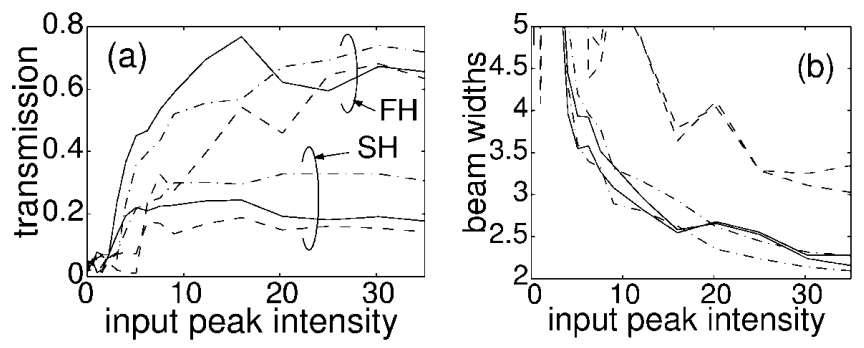

FIG. 5. (a) Transmission of the FW, $|w(0, L) / w(0,0)|^{2}$, and the $\mathrm{SH},|v(0, L) / w(0,0)|^{2}$, vs input peak intensity $|w(0,0)|^{2}$ of the FW. (b) Output beam width vs $|w(0,0)|^{2} . \quad L=100$, $\beta=G_{1,2}=50.83$ (solid lines), $\beta=G_{2,4}=101.66$ (dashed lines), and $\beta=G_{2,3}=82.25$ (dotted-dashed lines).

the generation of localized modes for varying strength of nonlinearity, i.e., a quasiperiodic soliton is generated only for sufficiently high amplitudes. This is of course a general phenomenon also observed in many nonlinear isotropic media. However, here the self-trapping occurs when the length scales of nonlinearity and fluctuations are comparable and, for quasiperiodic waves, with the quasiperiodicity being preserved in the variation of the amplitude of both components of the soliton.

Numerical simulations for other values of the phase mismatch $\beta$ reveal the same basic property of quasiperiodic self-trapping: Spatial solitons are formed in Fibonacci quadratic nonlinear slab waveguides above a certain power threshold, and such solitons are always quasiperiodic; i.e., they exhibit large-amplitude oscillations along $z$, which are composed of mixing of the two incommensurate Fibonacci wave numbers $\kappa_{1}$ and $\kappa_{2}$. The amplitude and width of these solitons depend on the difference between the phase mismatch $\beta$ and the nearest strong peak $G_{m, n}$ in the Fibonacci spectrum.

Finally, we would like to emphasize that the phenomenon described here is qualitatively different from the propagation of topological and nontopological kinks in disordered and quasiperiodic nonlinear media [4]. Such kinks can be well approximated by an effective structureless particle, which either preserves identity, as in the case of topological kinks [14,15], or decays rapidly into radiation [16].

In conclusion, we have analyzed SHG, self-focusing, and nonlinear beam propagation in Fibonacci optical superlattices with a quadratic nonlinear response. We have predicted spatial self-trapping of quasiperiodic waves and the formation of quasiperiodic envelope solitons which propagate for more than one hundred diffraction lengths without any significant loss of power. Such solitons have a localized envelope that traps the random-phase compo- nents through the phase- and frequency-locking effects of strong nonlinearity, and whose amplitude undergoes clearly detectable quasiperiodic oscillations. The results presented here would allow one to extend the concepts of self-localization and self-modulation of nonlinear waves to a broader class of spatially inhomogeneous media, and can also be found in systems of different physical context.

The authors acknowledge support from the Danish Technical Research Council (Talent Grant No. 9800400), the Danish Natural Science Research Council (Grant No. 9600852), and the Department of Industry, Science, and Tourism (Australia).

[1] See, e.g., Future Directions of Nonlinear Dynamics in Physical and Biological Systems, edited by P.L. Christiansen et al., NATO ASI Ser. B, Vol. 312 (Plenum Press, New York, 1993).

[2] A. Hasegawa, Phys. Fluids 18, 77 (1975); Phys. Fluids 20, 2155 (1977).

[3] M. Mitchell, Z. Chen, M.F. Shih, and M. Segev, Phys. Rev. Lett. 77, 490 (1996); see also M. Mitchell and M. Segev, Nature (London) 387, 880 (1997); Z. Chen, M. Mitchell, M. Segev, T. H. Coskun, and D. N. Christodoulides, Science 280, 889 (1998).

[4] See, e.g., S. A. Gredeskul and Yu. S. Kivshar, Phys. Rep. 216, 1 (1992), and references therein.

[5] Yu.S. Kivshar, S.A. Gredeskul, A. Sánchez, and L. Vázquez, Phys. Rev. Lett. 64, 1693 (1990).

[6] V. A. Hopkins, J. Keat, G. D. Meegan, T. Zhang, and J. D. Maynard, Phys. Rev. Lett. 76, 1102 (1996).

[7] M. M. Fejer, G. A. Magel, D. H. Jundt, and R. L. Byer, IEEE J. Quantum Electron. 28, 2631 (1992).

[8] D. Shechtman, I. Blech, D. Gratias, and J. W. Cahn, Phys. Rev. Lett. 53, 1951 (1984).

[9] W. Gellermann, M. Kohmoto, B. Sutherland, and P.C. Taylor, Phys. Rev. Lett. 72, 633 (1994).

[10] S. Zhu, Y. Zhu, Y. Qin, H. Wang, C. Ge, and N. Ming, Phys. Rev. Lett. 78, 2752 (1997).

[11] S. Zhu, Y. Zhu, and N. Ming, Science 278, 843 (1997).

[12] C. Balslev Clausen, O. Bang, and Yu.S. Kivshar, Phys. Rev. Lett. 78, 4749 (1997).

[13] See, e.g., Yu.S. Kivshar, in Advanced Photonics with Second-Order Optically Nonlinear Processes, edited by A. D. Boardman et al. (Kluwer, Amsterdam, 1999), p. 451.

[14] S. A. Gredeskul, Yu.S. Kivshar, L. K. Maslov, A. Sánchez, and L. Vázquez, Phys. Rev. A 45, 8867 (1992).

[15] F. Dominguez-Adame, A. Sánchez, and Yu.S. Kivshar, Phys. Rev. E 52, 2183 (1995).

[16] See, e.g., M. Hörnquist and R. Riklund, J. Phys. Soc. Jpn. 65, 2872 (1996). 